gueur, de diamètre et d'épaisseur variables, selon les positions respectives du mandrin et des enveloppes.

Pour le moulage, on dispose le moule sur une aire plane, et, parallèlement à lui, une voie portant sur uu truc de wagonnet la gamate dans laquelle on prépare le mortier composé de $800 \mathrm{kgs}$ de ciment mi-prompt, par mètre cube

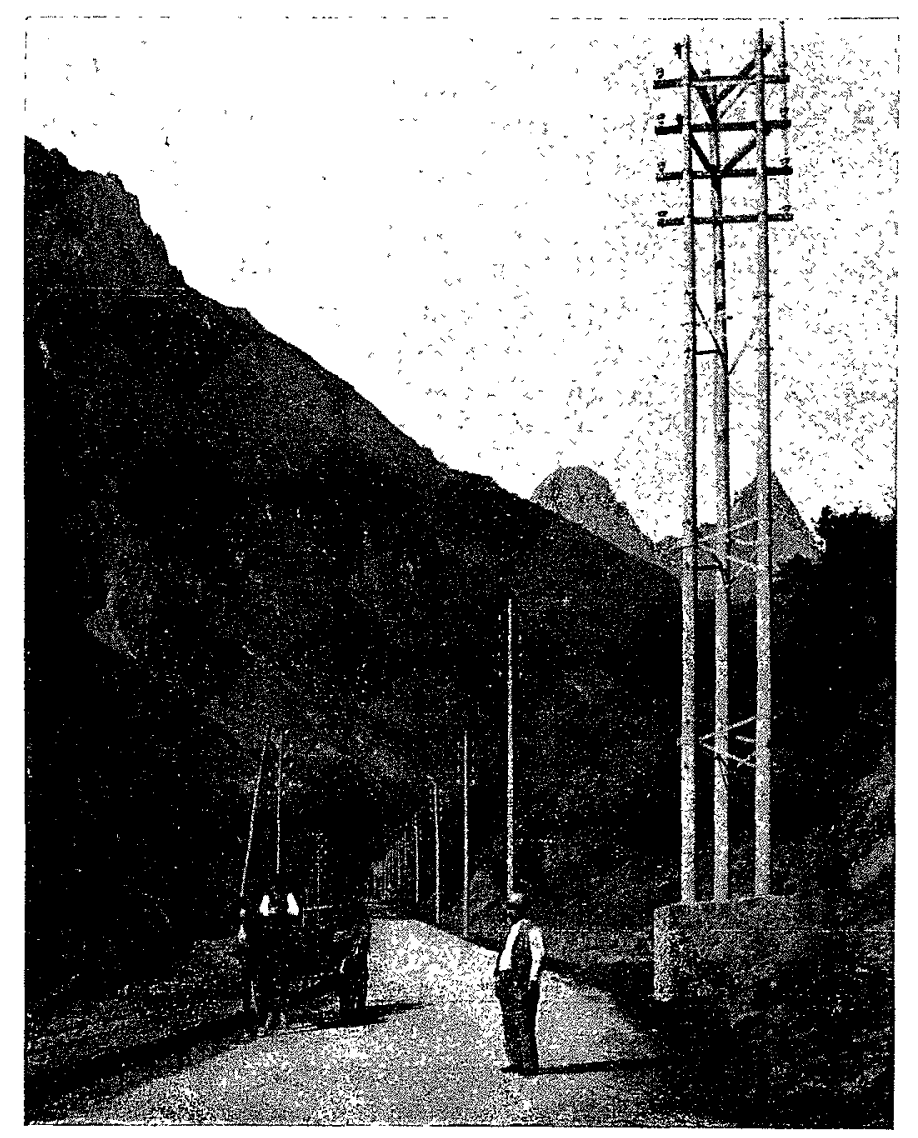

FIG. 4. - Vue de la ligne Livet-Grenoble, monlée sur poteaux Bourgeat.

de sable grenu. On peut ainsi faire deux ou trois coulées successives qui font parfaitement corps entre elles.

On emploie exclusivement pour cette tabrication un mortier composé de $800 \mathrm{kgs}$ de ciment naturel mi-prompt du rocher de Comboire.

Pylônes métalliques. - La Société Energie du SudOuest exposait une réduction au $1 / 5$ d'un pylône de $15 \mathrm{~m}$, en cornières d'acier, prévu pour supporter, avec des portées moyennes de $80 \mathrm{~m}$., une ligne triphasée primaire à 55000 volts, une ligne triphasé secondaire à 13000 volts, et une ligne de téléphone, pour le transport dans la région Bordelaise de l'énergie hydraulique produite dans l'usino de la Tuilière, sur la Dordogne, près de Bergerac. Ce type de poteau est identique à celui qui est employé par l'Energie Electrique du Littoral méditerranéen.

La maison BouCHAYER ET VIALLET. de Grenoble, exposait une maquette donnant en relief la gorge de Genissiat, telle qu'elle sera lorsqu'on aura aménagéé le Rhône en cet endroit. On y voit notamment le grand barrage et l'usine hydroélectrique dont la description ont été donnée dans $L a$ Houille Blanche de mars 1907. Des pylônes, en profilés d'acier, sont représentés supportant une double ligne qui part de l'usine pour se diriger sur Paris.

La Maison Joya père et fils, de Grenoble, exposait des poteaux métalliques de leur fabrication, pour hauts voltages, ainsi que des supports de ville pour lampes à arc.

M. P.

Nous rappelons que tout ce qui concerne la Rédaction doit être adressé au rédacteur en chef, $\mathbf{M}$. COTE, 24, rue Sully, a LYON, et que tout ce qui concerne l'Administration doit être adressé aux éditeurs, MM. GRATIER et REY, 23, Grande Rue, à GRENOBLE.

\section{USINES HYDRO-ÉLECTRIQUES DU MICHIGAN Transport d'énergie à 110000 volts}

La Grand-Rapids \& Muskegon Power $C^{\circ}$ a installé, dans la région de Grand-Rapids et de Muskegon (Michigan), un réscau de distribution électrique à très haute tension qui est alimenté par deux grosses usines hydro-électriques à basses chutes, installées sur la Muskegon River, et par deux autres usines de moindre importance, sur la Flat River près de Lowell. Son réseau est en outre relié à deux usines à vapeurde 2000 et $1500 \mathrm{HP}$ appartenant à des sociétés filiales. Elle possède en outre des droits d'eau qui lui permettront de produire, un jour, un total de $60000 \mathrm{HP}$.

Cette Société est de plus intéressée dans la Common. wealth Power $C^{\circ}$, de Jackson (Michigan), qui possède une grosse usine sur la Grand River, et six autres de moindre importance sur la Kalamazoo River.

Usine de Groton. - La plus importante de ces usines est située à Croton, sur la Muskegon River, qui a un débıl relativement régulier. Elle utilise une chute de $12^{\mathrm{m}} 20$ créée par un barrage, et peut développer normalement $10000 \mathrm{HP}$ Le barrage a une longueur de $183 \mathrm{~m}$., dont $78 \mathrm{~m}$. sont cons. titués par un barrage creux en béton armé formant déver. soir, et $61 \mathrm{~m}$. par une digue en terre. Entre ces deux élé nents distincts se trouve l'usine génératrice qui occupe $49 \mathrm{~m}$. de longueur. Du côté du déversoir. les coteaux qui limitent la vallée émergent légèrement au-dessus de la retenue, mais, du côté opposé, ils sont bien plus élevés, aussi en a-t.on profité pour construire la digue en terre par le système de remblayage à l'eau $\left(^{\star}\right)$.

I.a partie du barrage qui forme déversoir est constituée par un barrane creux en ciment armó, muni de hausses molbiles du type des vannes TAINTER, qui sont constituées par un segment de cylindre à axe hu rizontal, de $4^{\mathrm{m} 2} 27 \mathrm{de}$ raynn. En faisant tourner ce segment, on livre passage à I'eau à la partie inférieure. Cette eau tombe dans un bassin amortisceur qui est créé par un second déversoir, situé á $16 \mathrm{~m}$. à l'aval du premier. et qui est arracé à $2 \mathrm{~m} 29$ au. dessus du fond de ce bassin (Voir figrure 1). Il y a 8 de ces vannes, de $6 \mathrm{~m}^{10}$ d'ouverture chacune, réparlies par 4 de chaque côté d'un déversoir central de $12 m 20$ de largeur destiné à livrer passage aux bois de flottage et aux matières charriées à la surface, notamment aux glaces.

La pente de ce déversoir central, du côté aval, est de 2/3. L'ensemble est, continué par une dalle générale en ciment armé s'étendant jusqu'à $61 \mathrm{~m}$ : à l'aval du bassin amortisseur, de manière à éviter les affouillements, le sous-sol étant constitué par de l'argile dure sur laquelle repose de l'argile ordinaire et des alluvions, sables et graviers.

Les vannes TAINTER sont levées au moyen de chaines, attachées à leur partie inférieure, qui s'enroulent sur un treuil mobile actionné électriquement: Ce treuil est porté par un charriot qui se déplace le long du barrage en rou lant sur un pont de service, jeté au-dessus des vannes. Ce pont s'appuie sur les bajoyers qui séparent deux vannes consécutives et portent leurs axes de rotation: Le moteur, d'ure puissance de $4 \mathrm{HP}$, permet d'exercer sur chacune des chaines un effort de 7,2 tonnes.

L'extrémité inférieure de chaque vanne est munie d'ull madrier de chêne, qui vient appuyer sur un seuil en bois, disposé dans une rainure ménagée à la partie supérieure du déversoir. Pour assurer l'étanchéité contre les parois des bajoyers, on a disposé des handes de caoutchouc, de $10 \mathrm{~cm}$. d'épaisseur, qui entourent les extrémités des vannes et viennent s'appuyer contre les parois des bajoyers.

Le déversoir central est muni d'une hausse mobile d'un

(*) Les renseignements auxquels nous avons puisé pour cet article sont tirés de l'Engineering Record des 19 et 26 octobre 1907. 
autre type (bear trap). Celle-cl est constituéc par un battant de 1 in 83 de large et de $12 \mathrm{~m} 20$ de long, qui tourne autour d'un axe horizontal disposé à son extrémité inférieure amont (analogue à la partie $\mathrm{O} \mathrm{P}$ de la figure 3 ). Ce battant est relié à l'axe tous les $1 \mathrm{~m} 83$ par des coussinets, et l'axe est lujmême maintenu en place par des coussinets fixés sur la créte du déversoir central, avec vis de réglage pour assurer l'alignement. Le battant est levé au moyen de chaînes qui s'enroulent sur un treuil.

La digue en terre a une épaisseur de $6 \mathrm{~m}$. au sommet, et ses parements sont inclinés suivant un talus variant de 2,5 a 6 de base'pour 1 de hauteur à l'amont, et de 2 à 4 de hase par 1 de hauteur à l'aval. Elle est renforcée par un mur central en béton armé, de 0 305 d'épaisseur, dont laxe coincide avec la ligne d'intersection de la digue et du plan d'eau normal, et qui dépasse de $0^{\text {m60 }}$ ce plan d'eau.

Le remblayage hydraulique de cette digue s'est fait de la facon suivante : De l'eau sous pression, obtenue au moyen de pompes, était lancée contre la partie supérieure de la

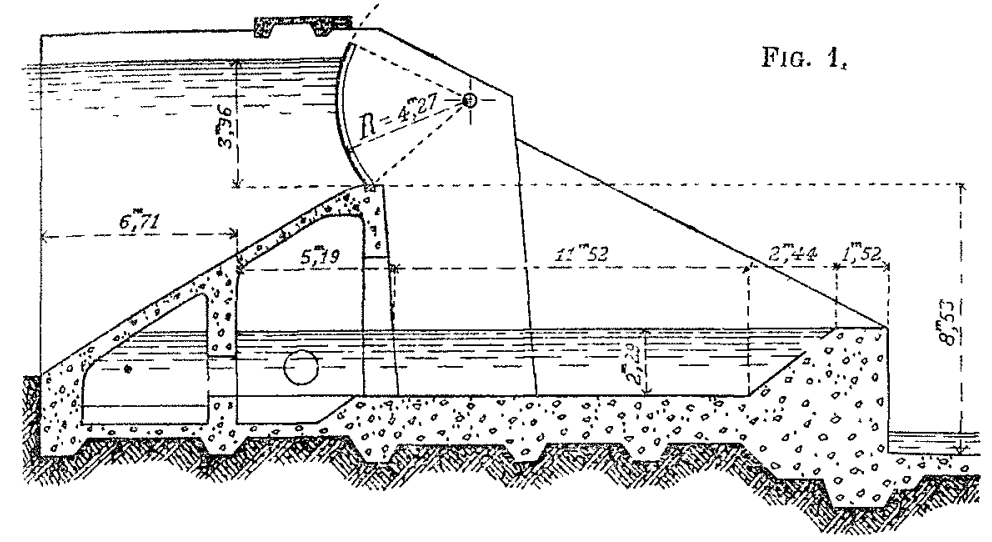

colline avoisinante, de manière à faire ébouler certaines parties. Ces parties éboulées étaient ensuite entrainées par un courant d'eau au moyen d'une canalisation à l'air libre, inclinée de 7 à 9 pour 100, aboutissant sur l'axe de la digue. Les matériaux terreux en se déposant formèrent la digue. La dépense s'est ćlevée à 44,5 centimes par mètre cube de digue, celle-ci cubant $80000 \mathrm{~m}^{3}$. La dislance maxima de transport etait de 244 mètres.

L'usine génératrice est établie en forme de $\mathbf{L}$, dont la petite branche est parallèle an cours de la rivière et con tient le matériel électrique, tandis que la grande branche, qui est dans l'axe du barrage, est réservé aux turbines. Celles-ci, du type Samson, sont au nombre de 8. Elles sont doubles, centriuètes parallèles, et à aspiration. Elles sont groupées par 4 , les unes à la suite des autres, de manière à former deux unités. Leurs roves mobiles, qui ont 1 in37 de diamètre, sont calées sur un même arbre horizontal, de $33 \mathrm{~m} 54$ de longueur et de $305 \mathrm{~mm}$. de diamètre, qui tourne à 225 tours. Cet axe actionne l'induit d'un alternateur Westinghouse, de $3600 \mathrm{kw}$., qui produit du courant triphasé sous 6600 volts, 30 périodes. L'excitation est assurée au moyen d'une dynamo de $45 \mathrm{kw}$. sous 125 volts, monté sur l'arbre commun. La régulation de la vitesse du groupe ainsi constitué est assurée par deux régulateurs, qui commandent chacun deux turbirues, mais qui sont disposés pour marcher solidairement.

Le bâliment des turbines est divisé en 4 compartiments parallèles au cour's de la rivière, dans chacun desquels est logé l'une des turbines doubles de chaque unité. Les turbines n'ont pas de huche, le compartiment supérieur, sur lequel elles sont fixies, formant chambre d'eau. Des vannes d'arrêt, placées à l'entrée des chambres d'eau, et à la sortie des galeries de fuite, ainsi que des joints ménagés au passage des arbres d'un compartiment à l'autre, permettent d'isoler chacun des compartiments en cas de reparations.

Les alternateurs ont élé prévus pour n'avoir pas une chute de tension de plus de 8 pour 100 lorsque l'on passe d'une charge nulle la pleine charge normale. Les rendements garantis sont de 0,94 à $1 / 2$ charge, 0,95 a $3 / 4$ de charge, 0,96 à pleine charge et 95,5 à 50 pour 100 de surcharge. La tension est élevée de 6600 à 72000 et 100000 volts, au moyen de transiormateurs statiques de $3000 \mathrm{~kW}$, a bain d'huile et refroidissement par circulation d'eau.

De l'usine de Croton partent deux lignes à 72000 volts, et une ligne à 110000 volts. L'une des premières sert de lien entre cette usine et celle de Big-Rapids dont il sera parlé plus loin ; l'autre va a la sous-station de Casnovia, distante de $35 \mathrm{~km}$. De cette sous-station, partent deux autres lignes allant, l'une à Muskegon City, à $40 \mathrm{~km}$. de là, et l'autre à

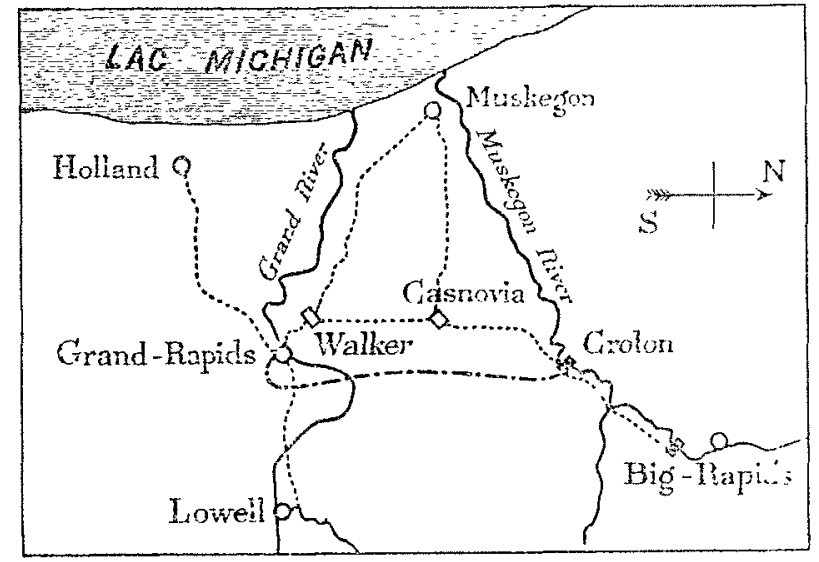

FIG. 2.

Grand-Rapids, à $34 \mathrm{~km}$. Un pont d'équilibre, de $35 \mathrm{~km}$., relie Muskegon à Walker, près de Grand-Rapids. De cette dernière ville part une ligne qui va jusqu'à Holland, à $31 \mathrm{~km}$.

Les lignes a 72000 volts sont constituées par 3 fils de cuivre qui sont altachés, suivant les sommets d'un triangle de 1 m83 de côté, sur des isolateurs Locke de $457 \mathrm{~mm}$. de hauteur et de $360 \mathrm{~mm}$. de dianètre. Ces isolateurs sont montés aux extrémités de deux bras en bois fixes aux poteaux. Le bras supérieur porte un fil de ler qui est mis à la terre tous les 5 poteaux. Ceux-ci, qui sont en cèdres du Michigan ou de l'Idaho, ou en cyprès des Etals du Sud, onl de $13^{\text {m }} 75$ a $18^{\mathrm{m}} 30$ et sont espacés de $40 \mathrm{~m}$. environ.

La lirrne à 110000 volts relie directement lusine de Croton à la sous-station de Grand-Rapids, et suit un parcours de $56 \mathrm{~km}$., absolument distinct de celui des lignes à 72000 volts (trait mixte de la carte fig. 2 ).

Les isolateurs sont d'un type spécial, de la General Electric $\mathrm{C}^{\circ}$, et sont analogues à ceux qui sont représentés page 78 . Ils sont formés de 5 cloches, de $254 \mathrm{~mm}$. de diamètre, suspendues les unes aux autres, à des distances de $204 \mathrm{~mm}$, par des anneaux formés de 4 ou 5 tours de fils d'acier de $3 \mathrm{~mm}$. de diamètre (1). Cetle ligne est montée sur

(1) Ce type d'isolateur est une modification du type de la figure $\alpha$ co contre, qui a élé d'abord préconisé par la General Electric Co. Ce modèle présente lavan-

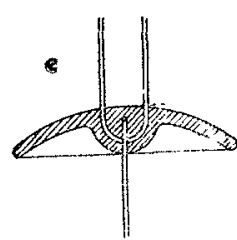

Hig, a.

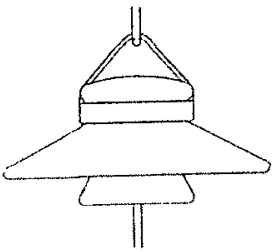

lig $s$ tare d'employer rlesimples cloches d'une fabrication cconomique, mais, par contre, il offre cot inconvénent gue les deux trous semi-circulaires, dont il fout percer chaque cloche pour le passage des anneaux de suspension, sont nicessairemont ì une as sez fable distance l'un de l'autre, ce yul dimınue la securité de l'isolement. La figure $\beta$ représente un autre type à suspension, construil par la Locke Insulator Manufacturing $C^{\circ} 11$ est constitus par des foubles cloches suspendues les unes aux autres par une lige rerticale fxée à la cloche interne et par une bride horizontale serréc autour de la partie supéricure de la cloche externe. 
des tours métalliques triangulaires, munis de troia bras auxquels sont sucpendus les i isolateurs comme le montre la figure 6 de la pagre 69 . La hauteur normale de ces tours est de $16^{\mathrm{m}} 16$ (53 pieds). I a hauleur du bras inférieur au-dessus du sol de $13^{\mathrm{m}} 31\left(43^{\prime \prime}\right)$ : celle du point le plus has des fils est de $12 \mathrm{~m} 20\left(40^{\circ}\right)$. La distance entre fils est de $2 \mathrm{~m} 436$. Les tours sont distantes les unes des autres de 161 mètres.

Cette ligne a été mise en service le 18 juillet 1908 et fonctionne, parait-il, très bien. Le courant de charge ne conduil qu à une perte à vide de 20 à 25 kilowatts $(\star)$.

Usine de Big-Rapids. - Cette usine est située à $29 \mathrm{~km}$. au dessus de la première, à Roger's-dam, près de BigRapids. La chute, qui est créée par un barrage, à une hauteur utile de $12 \mathrm{~m} 20$. Le débit varie de $20 \mathrm{~m}^{3}$ environ en septembre, à un peu plus de $150 \mathrm{~m}^{3}$ au printemps.

Le barrage a une longueur de $244 \mathrm{~m}$. et est constitué principalement par une digue en terre, saut à l'une de ses extrémités où se trouve une partie en béton armé, de $46 \mathrm{~m}$. de longueur, et à l'emplarement de l'ancien lit de la rivière où l'on a installé l'usine génératrice. L'inclinaison des talus de la digue est de 1/2 a l'aval, et de 1/3 à l'amont. La partie en béton armé est tout à fait analogue à celle de Croton. Elle comporte 6 vannes TAINTER, de $6^{\mathrm{m}} 10$ d'ouverture, réparties par 3 de chaque côté d'un déversoir central, qui n'a ici que 1 m 83 de largeur. Comme à Croton, l'eau qui s'écoule des vannes TAINTER, tombe dans un bassin amortisseur.

L'usine génératrice contient deux groupes électrogènes, composés chacun d'un alternateur Westinghouse triphasé de $2250 \mathrm{kw}$, à 6600 volts, 30 périodes, directement accouplé $\dot{a}$ un groupe de 2 turbines doubles, du type Samson, de 1 m37 de, diamètre, tournant à 225 tours par minute Une dynamo de $39 \mathrm{kw}$, montée sur l'arbre commun, assure l'excitation. I a régularisation de la viresse de chaque groupe est assurée par un régulateur Lombard, et celle du voltage, par un régulateur Tirrill(1). La tension est élevée à 72000 volts par 3 transformateurs à bain dhuile, avec refroidissement par circulation d'eau, et le courant est envoyé sous ce voltage, d'une part à l usine de Crolon, et dautre part à la ville de Big-Rapids.qui se trolıve à $8 \mathrm{~km}$. en amont.

Lorsque l'usine a été terminée, les groupes électrogènes ont été essayés sous la direction du professur Gardner Williams, de l'Université du Michigan. La partie électrique des essais a été faite par les « Electrical Testing Laboratories 》 de New-York. Les débits ont été mesurés au moyen d'un déversoir d'après la méthode Francis (2).

Usine de Lyons. - Cette usine utilise une chute de $7 \mathrm{m93}$, de la Grand River, à $30 \mathrm{~km}$ en amont de Lowel, et alimente le réseau de la Commonuealth Power $C^{\circ}$, en parallèle avec 6 autres usines installées sur la Kalamazoo River. Le débit minimum à Lyons est de $5,6 \mathrm{~m}^{3}$ environ.

Le barrage est entierement en béton armé, sauf une petite portion à l'une des extrémités. Il est du même type que celui du Croton, et est constitué par 10 vannes TAINTER, de 6 m10 de largeu" chacune, établies sur un déversoir, en béton armé, muni d'un bassin amortisseur, et réparties par 5 de chaque côté d'un déversoll central de $18^{\mathrm{m}} 30 \mathrm{de}$ Iargeur, destiné à évacuer les corps flottarts.

Ce déversoir central est surmonté d'une hausse mobile constiluée par un battant $O P$, qui a $2 \mathrm{~m} 44$ de longueur et, qui peut se lever de manière à relever de plan d'eau de

(*) Ces derniers renseignements sont tirús de l'Eleclrical World du 4 tévier 1909.

(1) Voir La Houille Blanche de juin 1907.

(2) La formule Francis, universellement employée aux Etats-Unis, est la suivante:

$$
Q=0.4152 \sqrt{2 g}[L-0.10 n H] H^{\frac{3}{2}}
$$
$n$ est le nombre de contractions latérales, $L$ la largeur et $H$ la hauteur
de l'eau sur le deversoir.
1 m52 au dessus de l'arrête du déversoir. Ce battant est relevé, comme celui de Croton, au moren de chaines attachées aux deux extrémités dune poutre $P$, de $1 \mathrm{~m} 06$ de hauteur, maintenue constamment verticale au moyen d'un syatème de guidage à articulation représenté sul la figure 3 . Un contre poids $\mathrm{C}$ de 9 tonnes, agissant à chaque extrémite sur le treuil de manceuvre du battant, diminue l'effort nécessaire à sa mancuvre.

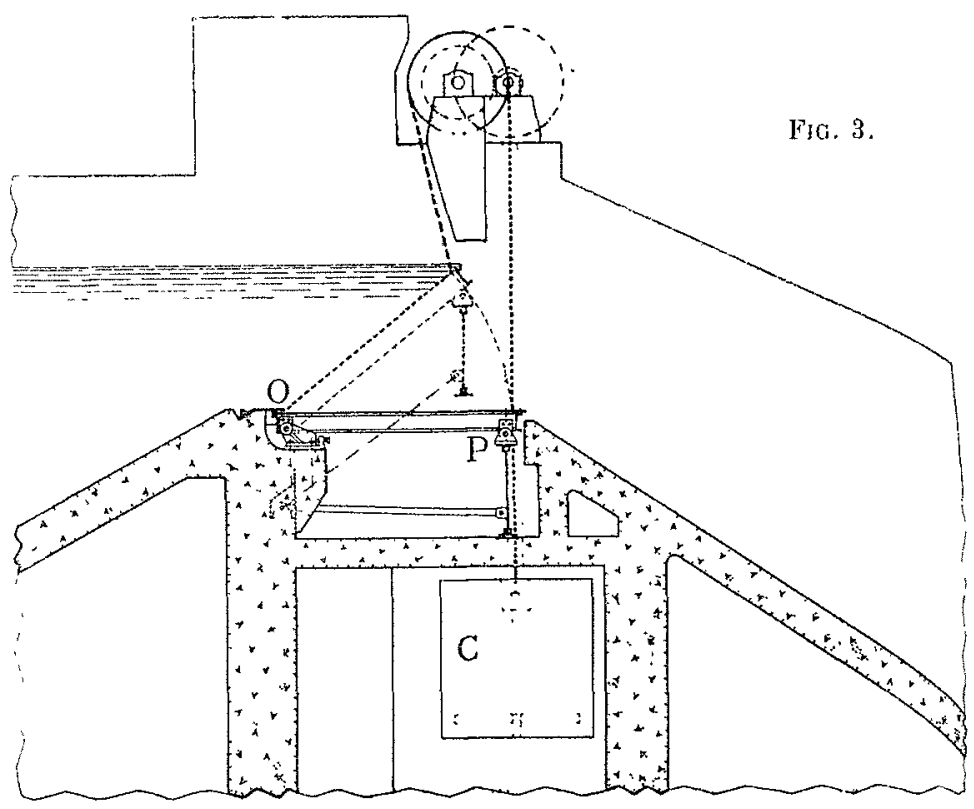

L'usine génératrice ect disposée absolument comme à croton. Elle ne contient qu'un groupe électrogène de $2590 \mathrm{kw}$., composé d'un alternateur triphasé de la General Electric $C^{\circ}$, à 6600 volts, 30 périodes, qui est actionné par un groupe de 4 turbines doubles de $1 \mathrm{~m} 27$ de diamètre, montées sur un même arbre de $42 \mathrm{~m} 39$ de longueur.

La tension est élevée à 72100 volts, et le courant est envoyé sous ce voltage à Jackson et dans les environs.

H. B.

\section{LA GALE DU CIMENT}

Les dermatologistes ont signalé un certain nombre d'affections cutanées d'origine professionnelle qui ont des caractèles bien particuliers.

Tel est le cas de la dermatose qu'on a étudiée chez les ouvriers en ciment; rare autrefois, elle est devenue plus fréquente depuis une dizaine d'années que les travaux en ciment ont pris une notable extension. Les constructions de tout genre en ciment armé qui se font maintenant partout ont muliplié les ocrasions de cette maladie protessionnelle, que les ouvriers ont dénommée la gale du ciment.

C'est une éruption qui se produit le plus communément aux mains, aux avant-bras, sur la poitrine, quand les ouvriers travaillent le corse nu, et parfois au visage. Elle affecte la forme de petites papules de la grosseur d'une tête d'épingle qui s'agrandissent du fait des grattages provoqués par le prurit intense qu'elles occasionnent. Elle ressemble de tous points à la gale vraie, sauf le sillon caractéristique du siège du sarcopte parasite ; comme elle, elle s'observe dans les espaces interdigitaux, dans les plis cutanés. Si les papules sont nombreuses, il peut se produire une véritable poussée d'eczéma ; l'exagération des sécrétions cutanées, de la sueur pendant les temps chauds, provoque facilement et l'apparition de la maladie et les complications.

Cette dermatose est causée par le maniement du ciment, dont la composition permet de se rendre compte de la venue facile de ces irritations. Le ciment est un composé de chaux, de silice, de fer et d'alumine et, suivant les provenances, 